\title{
Le désordre des plages ou la difficile émergence d'un territoire à part, 1920-1940
}

\section{Christophe Granger}

\section{OpenEdition}

12 Journals

Édition électronique

URL : http://journals.openedition.org/tourisme/146

DOI : 10.4000/tourisme.146

ISSN : 2492-7503

Éditeur

Éditions touristiques européennes

\section{Édition imprimée}

Date de publication : 1 juin 2014

Pagination : 68-79

ISSN : 2109-5671

\section{Référence électronique}

Christophe Granger, «Le désordre des plages ou la difficile émergence d'un territoire à part,

1920-1940 », Mondes du Tourisme [En ligne], 9 | 2014, mis en ligne le 30 septembre 2015, consulté le

10 décembre 2020. URL : http://journals.openedition.org/tourisme/146 ; DOI : https://doi.org/

10.4000/tourisme.146

\section{(c) $(1)$}

Mondes du tourisme est mis à disposition selon les termes de la licence Creative Commons Attribution - Pas d'Utilisation Commerciale - Pas de Modification 4.0 International. 


\title{
Le désordre des plages
}

ou la difficile émergence

d'un territoire à part, 1920-1940

\author{
Christophe Granger \\ Doctorant, ATER à I'Université de Strasbourg \\ [granger.chris@dbmail.com]
}

Résumé. On a coutume d'inscrire la plage dans une histoire longue et gentiment apaisée. Or si, en tant que territoire liminal dévolu aux plaisirs du corps, elle s'invente bel et bien dans le cours du XIXe siècle, rien n'est plus faux que de l'imaginer par la suite soumise à un docile mouvement de démocratisation. II suffit d'observer les âpres luttes dont les plages françaises ont été le théâtre dans l'entre-deux-guerres pour douter de cette généalogie. Les nouvelles postures corporelles, les dénudations plus poussées que jamais et la morale de l'abolition estivale des normes sociales, qui réforment alors l'usage du territoire balnéaire, suscitent en effet, à travers tout le pays, une intense croisade morale, marquée par les bagarres, les insultes, les pétitions, mais aussi la mobilisation de l'Église et des autorités municipales. Ces épisodes oubliés viennent éclairer un moment décisif dans la généalogie du territoire balnéaire ; celui où "la plage" se voit unifiée dans ses usages, lestée de sens commun et dévolue à un genre bien particulier de pratiques corporelles qui, n'appartenant qu'au rivage, ont aussi pour particularité désormais d'en constituer la nature.

Abstract. We are in the habit of registering the beach in a long and quiet history. Yet if it has been invented during the 19th Century as a liminal territory devoted to the pleasures of the body, it is far from the truth to imagine that it has been then quietly democratized. The many fights occurring on the French beaches in the interwar period are enough to doubt of this genealogy. The new physical postures, the nudity more pushed than ever, and the seasonal suspension of moral standards, which all reform the practices of this territory, arouse an intense moral crusade marked by fights, insults, petitions, and the mobilization of the Church and the municipal authorities. These long forgotten episodes enlighten the historical moment when the beach was unified in its manners throughout the country and devoted to a very particular kind of practices which belong only to the bank and now constitute its nature. 
S i l'historien a un rôle, ce n'est pas de peupler le présent de figures disparues, ni même d'entretenir, à la façon du gardien de cimetière, les rutilants édifices mémoriels agencés par d'autres, mais bien plutôt d'aller contre les histoires spontanées par lesquelles une société cherche à se donner des certitudes communes, bien plutôt d'arracher des morceaux entiers du grand récit collectif pour le rendre à ses incohérences, à ses inachèvements et permettre ainsi à chacun de s'en saisir autrement. Or, à cette tâche interminable, le territoire balnéaire oppose par avance un singulier empêchement. Non pas tant en raison de ses allures d'espace immuable - après tout, les historiens en ont vu d'autres, les grottes, les montagnes ou les monadnocks. Si la plage bouscule le travail de l'historien, c'est tout au contraire parce que, désormais bien identifiée, isolée du reste des territoires, dotée d'un nom commun sous lequel se range sagement la multitude des formes et des usages auxquels elle peut donner lieu, on a coutume de l'inscrire dans une histoire, longue et belle, qui semble s'imposer d'elle-même.

Acte 1. Un immense massif de croyances, de peurs et de certitudes, ficelées ensemble par les Anciens, ont, de Horace à Descartes, enveloppé le rivage d'une viscérale détestation.

Acte 2. Quelque part entre 1750 et 1840, un immense basculement de savoirs et de sensibilités, où entrent à la fois les édits neufs de la géologie et l'émerveillement nouveau

\begin{abstract}
"Ill lui faut donc se risquer entre ces gens étalés le cul au soleil avec un caleçon dessus. Elle bégaya quelques pas sur le sable. Grâce à ses nombreuses dioptries, toutes ces anatomies ne lui apparaissaient que fort floues, ce qui lui évitait de détailler les phalles de messieurs et les mottes de dames, que ni d'Adam ni d'Ève elle ne connaissait..." Raymond QuenEAU, Les Enfants du Limon, 1938
\end{abstract}

des peintres, a peu à peu fait de la plage un lieu de délectations et de rêveries insoupçonnées jusque-là. Et c'est là, en ces quelques décennies décisives, nous dit Alain Corbin dans son fameux Territoire du vide (1988), que s'invente bel et bien ce territoire liminal et tout entier dévolu aux plaisirs du corps qui nous est encore à peu près familier ${ }^{(1)}$.

Acte 3. Il n'est pas bien difficile d'imaginer, à suivre ce fil historique bien rectiligne, que, au $\mathrm{XX}^{\mathrm{e}}$ siècle, la plage fut en toute logique le théâtre d'un mouvement continu de standardisation, de sophistication ou, comme on voudra, de démocratisation des usages ainsi "inventés" par d'autres, une fois pour toutes.

On sait, et de longue date, combien ces étagements du temps sont trompeurs, combien, s'ils flattent l'appétit moderne pour les histoires continues qui filent droit au travers des siècles et nous placent au terme d'une longue remue de civilisation, ils opèrent au mépris de la complexité des phénomènes qu'elles emboîtent. Car dresser ainsi la genèse de la plage, ce n'est pas seulement regrouper, sous un même nom, trompeur, des choses infiniment différentes dans le temps. C'est, bien plus encore, escamoter à bon compte le fatras des écarts, des incohérences, des moments de résistance mais aussi des luttes de pouvoir, secrètes peutêtre, mais parfois considérables, qui sans cesse ont travaillé la plage et dont son histoire est faite. "La création $d u$ monde, disait joliment Proust, n'a pas lieu au début, elle a lieu tous les jours."

C'est là, très exactement, que réside le point de départ de la présente exploration. Défaire l'histoire longue de la plage, pour y introduire des fragments d'incertitude. Faire une place, si l'on préfère, à la série des épisodes, plus ou moins violents, plus ou moins articulés entre eux dont les plages françaises ont été le théâtre durant l'entre-deux-guerres, et qui, pour dire les choses aussi simplement que possible, ont en commun de ne pas cadrer avec la vision bien instituée de ce territoire et des façons ludiques d'en faire usage. " $I l$ faut y aller plus doucement, presque bêtement. Se forcer à écrire ce qui n'a pas d'intérêt, ce qui est le plus évident, le plus commun, le plus terne”, préconisait Perec dans ses Espèces d'espaces (Perec, 1974). Voilà pour la démarche. Elle permet, à qui parcourt les plages des années 1920-1930, de prendre au sérieux un bouillonnant matériau qui a toute 
chance de se voir ravaler au rang d'anecdote piquante, aux marges, autrement dit, du savoir digne de ce nom. Insultes, bagarres, pétitions, intervention du maire, du curé, du député : dès lors qu'on accepte de leur chercher du sens, c'est une controverse nationale oubliée que dessinent ensemble ces fragments (Granger, 2009). Une controverse qui, par les enjeux qu'elle mobilise, permet d'éclairer l'histoire de la plage et des modalités changeantes de son appropriation. Entendons-nous bien : nous n'avons pas cherché, ici, à réhabiliter ces positions d'hostilité, ni même à rendre voix à ceux, conservateurs souvent, qui les faisaient vivre. Mais en les suivant, en soumettant autrement dit l'histoire de la plage à l'un de ces salutaires "exercices de lenteur" auxquels invitait il y a peu Patrick Boucheron (20।2), il devient possible de dévoiler, d'ordinaire imperceptible, le bouillonnement social particulier qui, à ce moment précis, a vu la plage devenir un territoire à part entière ; un territoire unifié d'un bout à l'autre du rivage sous un nom commun, et dévolu désormais à un genre bien particulier de pratiques, corporelles pour l'essentiel, qui, parce qu'elles ne peuvent tout bonnement pas avoir lieu ailleurs, ont aussi pour particularité d'en constituer désormais la nature. L'autonomisation d'un territoire, voilà bien ce qui est en jeu dans cette bataille oubliée de l'entredeux-guerres, dont les épisodes et les enjeux, moraux, nationaux et symboliques, ont à présent déserté les mémoires.

\section{"ILS MÉRITENT LA BASTONNADE"}

Aborder les plages d'entre-deuxguerres, c'est s'exposer à rencontrer un épais tissu de heurts et de violences dont on ne soupçonne plus la virulence. L'un d'eux, sans doute pas le plus net, pas le plus vivace, tout compte fait, peut avantageusement servir de point d'entrée dans ce moment singulier.

Quercy, 1934. Plusieurs semaines durant, un petit village, près de Cahors, est le lieu d'une intense passe d'armes dont l'écho, d'abord, ne dépasse guère les frontières $\mathrm{du}$ canton. Une bande de "vacanciers", plutôt jeunes, venus passer là une partie de leur été, ne tarde pas à investir la petite plage locale, improvisée depuis de longues années déjà le long de l'étang qui marque les confins de la commune. D'un coup, ils la mettent à l'heure inédite de pratiques balnéaires qui, pour être d'une fréquentation familière aux classes moyennes des villes en ces années, ont ici le parfum de l'insolite. Chaque après-midi, en effet, filles et garçons réunis en une inhabituelle camaraderie des deux sexes, se baignent, chahutent et flirtent dans des tenues, courtes et ajustées, qui laissent voir des cuisses, des dos, des bras laissés d'ordinaire aux jeux de l'imagination. Et de longues heures durant, allongés en public, ils se font bronzer au soleil. Contraints de déserter "leur plage", ceux du village ne tardent pas à s'offusquer. Un dimanche, alerté par le scandale qui couve, le curé s'en mêle, probablement enhardi par la condamnation des "costumes indécents" à laquelle se sont livrés les évêques de France quelques semaines plus tôt. Aux débordements de la plage, il n'hésite pas à consacrer son prêche. Face à ces dépravations qui "ruinent la paix des familles", dit-il, face à ce scandale qui défigure sa paroisse, et que "Dieu ne saurait absoudre", les vacanciers seront privés de sacrement le 15 août. L'affaire s'envenime. Les commerçants s'inquiètent. Et bientôt, le maire, habile d'abord à ramener le calme sur la place du village, doit céder aux insistances du curé. Quelques jours plus tard, sur le modèle de ceux qui foisonnent alors aux quatre coins du pays, il prend un arrêté municipal qui interdit les conduites inconvenantes sur la plage locale. La querelle s'éteint, les vacanciers décampent, et le petit étang retrouve la sagesse de ses usages passés ${ }^{(2)}$.

Que faire, en historien, de cette scène minuscule ? Il n'est pas bien difficile, dira-t-on, d'y voir une bizarrerie insignifiante, à peine un Clochemerle estival, au fond, qui, parce qu'il met en scène la résistance des traditions à l'ascension inéluctable des conduites modernes, ne mérite pas grand-chose de plus que de se voir reléguer dans le ventre anecdotique de la grande histoire des plages. Seulement, s'en tenir là, c'est se condamner à passer sous silence la multitude d'autres affaires nouées en ces années sur un mode similaire à travers tout le pays. Et c'est, surtout, renoncer à comprendre ce que, ensemble, elles énonçaient alors à mi-voix. 
Les plages et les pratiques qui les peuplent prolongent à leur façon les âpres luttes qui, à la faveur des crises sociales, morales et idéologiques, tenaillent alors une société française qui n'en finit pas de sortir de la guerre (Charle, 200 I ; Scot, 1988). La brutalité des mots dont elles sont le lieu, la vivace économie de la menace et de l'insulte sont ici à prendre au sérieux. "N'est-ce pas, sous le costume moderne, la vision des spectacles libertins qu'on reprocha aux grandes époques de décadence? Ces hommes nus, ces femmes sans sexe, cet étalage insolent, cette promiscuité sans pudeur fait songer à une partouze licite", s'emporte entre cent autres le Paris-Matinal, observant la plage de Biarritz en 1927. Même pour qui sait combien une sourde violence innerve toute la société de l'entre-deux-guerres, ces virulences ont de quoi surprendre. "Ils méritent la bastonnade, lit-on en 1932 dans un copieux article de La Nation, journal de la droite catholique. Le crime est qu'ils compromettent les qualités foncières de la race, de la décence, la pudeur, la modestie, la tenue, le sens $d u$ respect. Il est partout, des inconscients et des vicieux. Le grave est qu'ils soient, sur nos plages, en si grand nombre et s'étalent aussi cyniquement." Mais c'est sans doute dans le pamphlet nationaliste que Georges Anquetil consacre entièrement à la question, Le Bal sur le volcan (1927), que se dit le mieux l'hostilité. "Vermine, racaille, ramassis de métèques plus ou moins dorés, échantillonnage de ce que le monde entier comporte

\section{ILLUSTRATION 1}

Le Petit Journal ILUustré, 1927

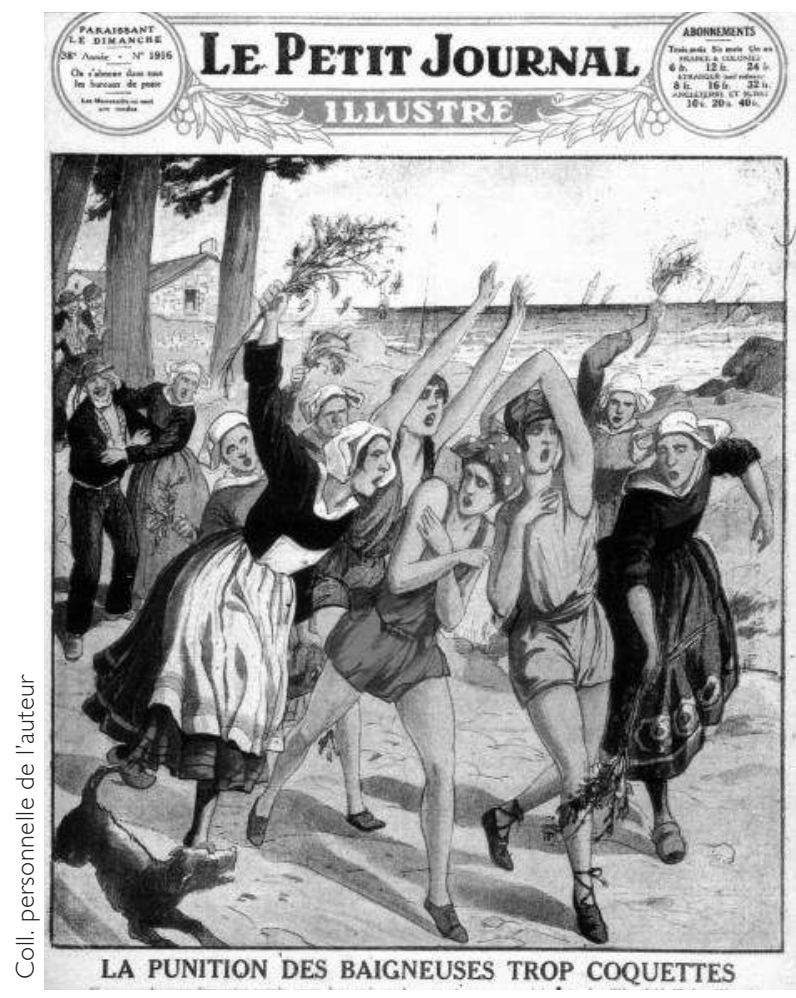

d'abject et d'inutile [...]. Ces dames n'auront plus qu'à montrer aux amateurs les muscles et les sphincters en plein travail, maintenant qu'elles ont laissé suffisamment contempler toutes leurs rotondités et tous leurs orifices au repos!" Des plages, photos à l'appui, Anquetil fait ainsi le territoire de toutes les abominations et celui, par la même occasion, d'une vaste déploration politique. "Ces spectacles, estime-t-il, sont révoltants et font partout des révoltés. Ils ne marquent pas la fin d'une société. Ils sonnent le tocsin d'un monde" (Anquetil, 1927).

Mais l'indignation qui se dessine ainsi ne va pas seule. Entre 1925 et 1935, en l'espace d'une décennie autrement dit, elle s'est doublée d'une série de collisions plus concrètes. Dans des localités souvent petites ou moyennes, une multitude de plages ont, en effet, accueilli des débordements locaux, où sont venus, pour un temps, se souder la réprobation dont s'accompagnaient les nouvelles conduites balnéaires. Souvent semblables, ces bagarres de plage sonnent comme la réitération des formes anciennes du chahut populaire. En 1927, près de Pléneuf, en Bretagne, une poignée de villageoises, excédées par les "tenues indécentes" qu'arborent les baigneuses sur la plage voisine, organisent une petite séance punitive. Un matin, munies de ronces et d'or- 
ties, elles se jettent sur les baigneuses pour les fouetter énergiquement ( $c f$. illustration 1). En 1933, à Malo-lesBains (Nord), puis -Atlantique) ou encore à Sanary-sur-Mer (Var), des groupes l'année suivante à Batz-surMer (Loire d'habitants et d'habitués opèrent de la même façon, improvisant sur "leur" plage des "corrections publiques". Après avoir parlementé, menacé, insulté, ils finissent le plus souvent par empoigner les vacanciers trop dénudés ou par les chasser à coup de pierres et de morceaux de bois. "Bravo!, félicite le curé de Batz dans son bulletin paroissial. Vous avez hué ces impudiques, vous les avez chassés. Bravo! Ne laissez pas le haut du pavé aux porcs et aux sauvages."

\section{LA "LUTTE CONTRE L'IMMORALITÉ DES PLAGES"}

Mal connus, difficiles à dénombrer tant ils demeurent inscrits dans l'exiguiité des existences locales, ces épisodes cinglants cachent en réalité l'émergence d'une mobilisation nationale bien mieux organisée. Et c'est elle qui donne sens à ce qui se joue sur les plages de France, ou plus exactement à ce qui, depuis les plages, se joue alors dans l'ordonnancement de la société française. À dater des années 1920, en effet, à travers tout le pays, une multitude d'acteurs se sont groupés, au sein d'associations locales d' "honnêtes gens”, ou au sein de plus influentes ligues de moralité, pour faire naître, au sujet du territoire balnéaire, une véritable croisade morale. Dirigée par un professeur de l'université de Strasbourg, la Fédération française des sociétés contre l'immoralité publique tient à ce jeu un rôle central. Vieille déjà de deux décennies, protestante en ses origines, puis résolument œcuménique (Le Naour, 2003), cette dernière, investie en ces mêmes années dans la moralisation du cinéma, est le lieu d'une intense activité qui vise à coordonner l'offensive. En son sein prennent forme un objet national d'indignation, bientôt baptisé "lutte contre l'immoralité des plages”, et le répertoire argumentaire propre à faire de celleci une priorité dans l' "assainissement $d u$ pays", mais aussi un plan d'action et le réseau d'acteurs disposés à le mener à bien ${ }^{(3)}$. Les hommes qui assurent le gros de cette mobilisation méritent attention. Médecins, avocats, commerçants, militaires, universitaires $^{(4)}$ : la plupart d'entre eux, s'il ne se perçoivent pas comme réactionnaires, appartiennent à la droite chrétienne; et surtout, ils proviennent pour l'essentiel de ces "classes moyennes" traditionnelles, provinciales et éduquées, dont l'influence sociale, économique et culturelle s'affaisse après la Grande Guerre, et qui, en ces années, sont portées à mobiliser la morale, et singulièrement la morale familiale, comme capital de substitution (Lenoir, 2003). Mais l'important, pour ce qui nous occupe, est que ces hommes s'emploient à faire des manières de plage une question d'intérêt public et une affaire de morale. Le dispositif est limpide. Ce qu'ils réprouvent ? Les conduites corporelles, déplorables à leurs yeux, dont le rivage est devenu le théâtre : dénudations, bains de soleil, décontraction des allures. Retournant mot à mot les édits neufs de la morale balnéaire, ils en font le lieu de prédilection où s'annoncent ensemble la déchéance de la morale privée et la ruine de la morale publique. Sous le dévoilement des corps, l' "attentat à la pudeur". Sous le bronzage, une "niaiserie dangereuse pour le pays". Sous la décontraction des allures, l' "esprit d'indécence". "J'ai même vu, affirme l'un des artisans de cette lutte balnéaire, ces individus se livrer, sur la plage, au simulacre de l'acte sexuel en présence de passants, de promeneurs, d'ouvriers, et les rires expliquaient assez que le jeu était intentionnel" (Pourésy, 1928). Et sous le silence des maires, la compromission des pouvoirs publics.

Les actions que mènent ces hommes témoignent de leur détermination. À lire l'abbé Pradel, qui en relaie l'action auprès des milieux catholiques du pays, leur plan de bataille observe une armature limpide : 1) "former les consciences", 2) "mettre en mouvement les autorités publiques", et 3) "réprimer les excès”. Inlassablement, dans les pages du Relèvement social, du Bulletin d'information antipornographique et de plus influents journaux nationaux et locaux, ils s'efforcent d'alerter l'opinion et de mettre en circulation les motifs de la lutte. Sur les plages, l'été, ils tiennent des meetings publics. Ils distribuent des tracts, organisent des “commandos" et mettent à la dis- 
position des "comités de salut" locaux une série de modèles de pétitions qui, à la manière de celle qu'a rédigée en 1934 le Groupement calaisien pour le relèvement de la moralité publique, sont destinées aux maires ou aux préfets : "Considérant que la plage de Calais est surtout une plage de famille, le Comité déplore le laisser-aller, signalé dans la presse locale, pendant les dernières saisons. Pour éviter ces inconvénients, il demande à M. le Maire de vouloir bien prendre un arrêté réglementant le port du costume de bain, le déshabillage à la plage, le port du peignoir, les bains de soleil, comme cela a été fait dans un grand nombre de stations balnéaires, à Biarritz, Bayonne, Deauville, Wimereux, Malo-lesBains, etc." (25 mai 1934). À leur suite, ces hommes parviennent, souvent sans mal, à mobiliser les offices de tourisme et, plus encore, en ces décennies travaillées par le net regain d'influence de l'Église catholique après la Grande Guerre (Nord, 2003 ; Wardhaugh, 2009), un clergé local tout disposé à s'engager dans la conduite nationale des choses morales. "Le temps est venu, expliquent plusieurs desservants de paroisse à l'été 1933, où doit cesser cette promiscuité qui est le triomphe de l'immoralité éhontée, et de ces complaisances pour le vice qui finissent par émousser des consciences qui hier encore étaient délicates" (rapporté dans La Croix, I 2 juillet 1933). À Arcachon, à Royan, à Boulogne, les "tournées de plage" des curés, soucieux de "résister à la vague de paganisme qui nous envahit", consti- tuent ainsi de véritables événements locaux.

Mais la mobilisation culmine, à vrai dire, à l'été 1934. Dans les événements sanglants du 6 Février, et les menaces qu'ils font d'un coup peser sur les institutions démocratiques, ses promoteurs trouvent sans difficulté l'éclatante confirmation de leur cause et l'occasion d'en enhardir le propos. "Les anciens combattants l'ont crié à Paris le 6 février, explique une affiche placardée sur les côtes en juillet 1934 : 'la France veut vivre dans la propreté.' Nous ne tolérerons donc pas que la plage soit déshonorée par des exhibitions et des jeux malpropres, qui constituent parfois de véritables attentats publics à la pudeur ${ }^{(5)}$ ”. À cet effet, des "pochettes-plage", contenant des modèles de plainte et de pétition, des textes de médecins condamnant le bronzage ou les dénudations trop poussées, et de grandes affiches à apposer sur le pourtour du rivage, sont produites et mises en vente à travers le pays. À la manière du texte que porte la Protestation des pères de famille contre les désordres de la plage (1934), la bataille est vive: "Le spectacle que nous offre à la plage une minorité d'excentriques des deux sexes est de plus en plus déplorable. Comme pères de famille, ayant charge d'âmes, Nous protestons contre ce scandale. Il ne s'agit pas de pruderie. Il s'agit de dignité humaine et de décence publique. La plage est à tout le monde, sans doute, mais elle appartient d'abord aux personnes correctes, qui sont la majorité et qui valent bien les autres."

\section{LES ARRÊTÉS MUNICIPAUX ET L'ORGANISATION DES CONDUITES BALNÉAIRES}

À bonne distance, on aurait tort d'imaginer que pareille lutte, vieille à présent de sept ou huit décennies, et qui s'est, comme on sait, révélée impuissante à imposer ses visées, n'a fait que se tenir sur le rebord anodin du territoire balnéaire. Les détails de cette croisade oubliée font, au contraire, toucher du doigt la vitalité des débats dont la plage est alors le lieu, et l'intensité de la discorde sociale qui s'anime alors à son sujet. Mais elle a aussi très concrètement pesé sur le façonnement de la plage, telle qu'on la connaît encore, sur les pratiques qui peuvent s'y déployer et sur le genre d'autorité qui peut s'y exercer. Car s'ils échouent à engager l'intervention de l'État, à laquelle ils aspiraient, et qu'ils n'ont eu de cesse de susciter, menant la question à plusieurs reprises sur le bureau du ministre de l'Intérieur, entre 1927 et 1932, et jusqu'au sein de la Chambre des députés, les "croisés" de la morale balnéaire ont en revanche réussi à se frayer un chemin jusqu'à l'oreille des maires.

En une dizaine d'années, d'un bout à l'autre du pays, les conseils municipaux ont habillé les plages de France, depuis celles du littoral jusqu'à celles, menues parfois, qui, dans les terres, ornent les rivières ou les étangs, d'un épais massif d'arrêtés municipaux. La moisson est impressionnante : deux à trois cents de ces textes singuliers voient le jour 
entre 1925 et 1935 ( $c f$. illustration 2). Pris dans le canevas infini des politiques locales et des formes traditionnelles de gestion communale de la plage, dont Johan Vincent a judicieusement montré les logiques (Vincent, 2007), ces dispositions réglementaires se révèlent toutefois suffisamment semblables pour laisser apparaître l'esquisse d'un véritable dispositif.

Ce que disent ces textes ? D'abord, que la manière de vêtir et de tenir son corps, en ce lieu, ne s'improvise pas. Tous interdisent, ainsi, les déshabillages et rhabillages sur la plage, y compris sous les tentes qui la jon- chent, "à moins, fait préciser le maire de La Seyne-sur-Mer, que ces tentes soient fermées et en toile suffisamment opaque pour que les personnes qui s'y déshabillent ou s'y rhabillent ne puissent en aucun cas être vues de l'extérieur" (juillet 1934). Tous prescrivent aussi une "attitude correcte", "discrète" ou "non provocante", et, "considérant que la tenue sur la plage provoque des réclamations dont certaines paraissent justifiées", ordonnent le port d'un "costume de bain décent”. À La Flotte (Charente-Maritime) et dans une multitude d'autres communes, "l'usage du slip ou caleçon court et

\section{ILLUSTRATION 2}

ArRêté municipal, Gournay-sUR-Marne, 18 JUILlet 1934

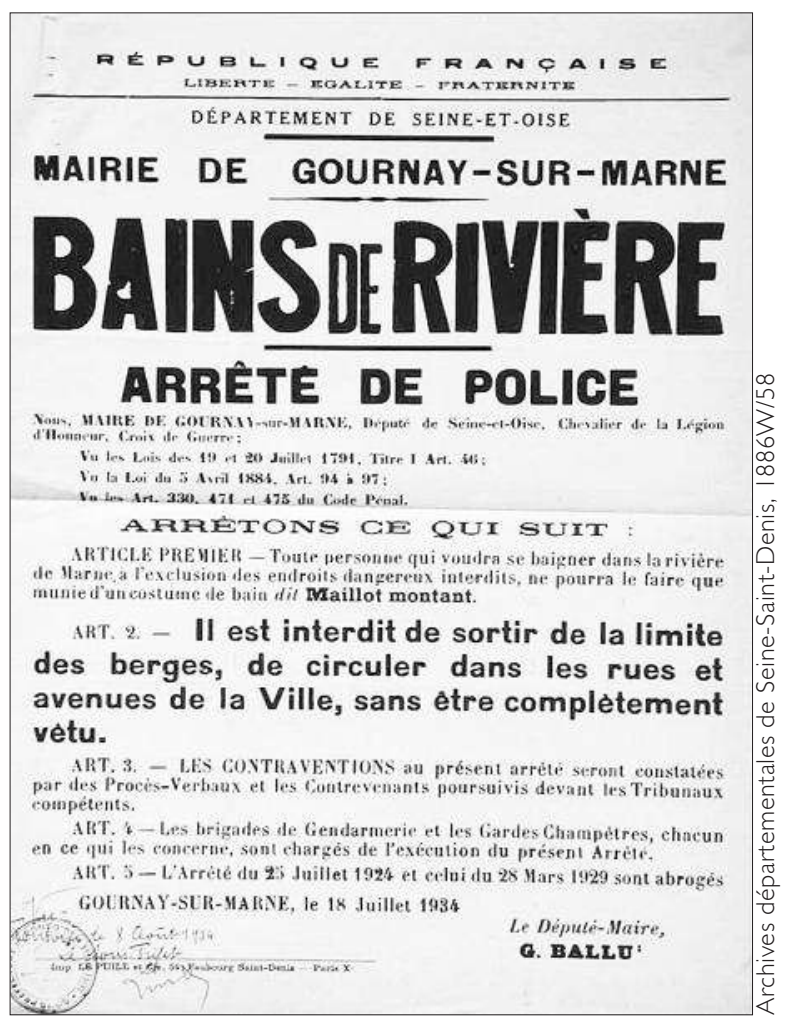

du maillot d'étoffe transparente est formellement interdit" (arrêté municipal, La Flotte, 17 août 1933). Certains maires, comme celui de La Rochelle, Léonce Vieljeux, se montrent d'une précision scrupuleuse sur ce chapitre, posant les contours d'une minutieuse arithmétique du nu. "Article 2. Il est interdit à toute personne de se baigner, de circuler ou de s'exposer sur la plage, même sous prétexte de cure d'héliothérapie, sans être revêtue d'un costume de bain complet, c'est-à-dire couvrant le torse, le bassin et la partie haute des membres inférieurs" (arrêté municipal, La Rochelle, juillet 1934). D’autres, à Calais, à Wimereux ou à Liverdun, interdisent de s'adonner, en maillot, aux "parties et jeux de ballon", et même de "circuler sur la plage, sans être enveloppé d'un peignoir ou de tout autre vêtement convenable constamment tenu fermé” (arrêté municipal, Calais, 24 juin 1934). Plusieurs municipalités, enfin, en Charente et dans les Landes, vont plus loin encore; elles réprouvent le bain de soleil, jugé "prétexte à des exhibitions inconvenantes”, et en relèguent volontiers la pratique aux "extrémités nord et sud de la plage, en dehors des villes, loin de la foule" (arrêté municipal, Mescher, 22 juin 1934).

L'efficacité de cette réglementation, autrement dit sa capacité à organiser les conduites balnéaires, ne se laisse pas commodément approcher. Des policiers embarrassés par leur tâche estivale, quelques contraventions et quelques vacanciers fâchés de se voir verbalisés, plusieurs 
recours devant les tribunaux, aussi, pour évaluer la justesse du pouvoir que se donnent ainsi les maires, et voilà à peu près toutes les traces qu'il nous reste. Mais, tout compte fait, ce qu'il y a de plus remarquable dans cette affaire, n'est pas là. S'en tenir à observer que ces batailles de plage échouèrent finalement à policer les mœurs balnéaires, ou qu'elles ne parvinrent jamais à ériger ce qui se jouait sur le rivage en problème d'État, c'est manquer l'essentiel : car elles posent bel et bien la question de la nature de ce territoire, en des années où, précisément, la définition juridique de la plage avoue ses embrouillements et ses lacunes (Bernard, 1930) et où elle est dotée, dans l'espace public, d'une figure métonymique qu'elle ne se connaissait pas.

\section{Naissance D'UN TERRITOIRE À PART}

Autant ne pas y aller par quatre chemins : si les réactions sont si brusques, l'inquiétude si grande, les positions si tranchées, c'est qu'en dix années à peine le territoire balnéaire est le lieu d'une métamorphose à ce point profonde qu'elle le rend littéralement méconnaissable. Et c'est bien de cette étrangeté nouvelle, de cette incapacité à faire tenir le territoire balnéaire et ce qui s'y joue désormais dans les définitions anciennes de la plage, celles, pour aller vite, qui avaient cours avant la Grande Guerre, que nous parlent avant tout ces virulents épisodes et ceux qui les animent. En creux, mais probablement mieux qu'une centaine de descriptions dépareillées, ramassées à droite à gauche, et vite ficelées ensemble au mépris des logiques qui les animent - ici la plage de Biarritz et ses allures de parc zoologique selon Le Figaro, là la photo des plaisirs du rivage méditerranéen érigé en front pionnier, là encore la littérature, neuve, des reportages de plage ou la typologie mi-sérieuse mi-rigolarde des mœurs balnéaires, etc. -, ces heurts passés donnent corps au portrait de la plage d'entre-deuxguerres. Pas question, ici, d'en reprendre le tableau, tant les choses sont connues. L'important est de conserver à l'esprit que ces figures balnéaires, si elles ne surgissent pas de rien, se sont imposées en l'espace de deux décennies. La plage, pour tailler au plus net, devient le lieu attitré d'une présence particulière des corps. Outre l'avènement $\mathrm{du}$ bronzage (Ory, 2008), âprement disputé dans les cénacles médicaux et finement codifié dans les milieux de la bourgeoisie cultivée - Marcel Aymé s'amuse, par exemple, de ceux qui s'échinent désormais à "être nègre cent pour cent, et même un peu plus" -, la plage commande, dès alors, d'arborer une silhouette, qui réclame un travail de préparation, et une apparence soumise tout entière aux codes de la "beauté $d u$ dehors” (Vigarello, 2004), c'est-à-dire à un moderne devoir de "naturel". Pas de maquillage, pas d'effet de coiffure, pas de sophistication. Elle réclame aussi l'habitude, nouvelle, d'une décontraction particulière des allures et des gestes, une aisance à l'alanguissement des postures qui, d'un coup, plongent la rectitude scrupuleuse de la vieille bourgeoisie dans l'anachronisme. Elle est enfin le lieu d'un décisif jeu avec la pudeur qui, s'il n'est pas entièrement neuf, atteint en ces années des sommets. Les maillots rétrécis et ajustés aux formes, qui livrent aux regards des cuisses, des fesses et des poitrines d'ordinaire inaccessibles, font $\mathrm{du}$ rivage le territoire attitré des dénudations licites, que l'horizontalité publique des corps, inconnue jusquelà, rehausse d'un trouble parfum de provocation. "C'est un grouillement de tons, de chairs, dans lequel les jambes et les bras s'affirment, nus, différemment hâlés, du cuivre au café au lait. Les jambes nues des jeunes femmes voisinant les jambes nues des jeunes hommes, les bras se touchant presque, dans une promiscuité sportive qui donne le sentiment d'une grande liberté d'allures, mais non ambiguë", s'enthousiasme en 1927 la très sérieuse Revne de Paris, appliquée, comme tant d'autres, à saisir sur le vif le nouveau paysage humain que proposent les plages.

À ces conduites nouvelles, fermement reliées à la définition sociale des vacances qui surgit après-guerre, on n'a pas manqué de donner une armature explicative. On sait que ces impudicités passagères n'ont rien de simples dévergondages nés du souci d'oublier la guerre. On sait aussi combien, sous l'absence apparente de règle qu'elle autorise, la plage recèle en réalité des règles bien plus serrées qu'à l'accoutumée. "Il n'y a pas de lieux plus convenable 
qu'une plage, pas d'endroit où l'on soit plus correct, s'amuse dès alors la presse satyrique. Comme on y est à peu près nu, le moindre geste équivoque risque de passer pour un attentat à la pudeur" (Le Rire, 22 juillet 1938). On a même pris pour habitude de traquer, en ces nouveaux usages, l'expression d'un exhaussement des disciplines, ou, si l'on préfère, d'une plus grande maîtrise de soi, dont Norbert Elias, raccourci oblige, est devenu le nom de passe à tout faire. À raison, sans doute. Car le sociologue, penché dans les années 1930 sur sa Civilisation des mours, n'avait pas manqué d'y inclure, en quelques pages décisives, l'observation des mœurs balnéaires de son temps. Et remarquant combien 'il est impensable qu'une fermme ait pu se montrer au XIX siècle en public, sans se faire conspuer par la société, dans un de ces costumes de bain qui sont aujourd'hui d'usage courant", il pouvait estimer que les nouvelles conduites de plage réclamaient un "niveau plus élevé de contrôle des pulsions” et qu'elles portaient par conséquent la marque d'une civilisation plus accomplie (Elias, 1973). Et de tout ceci, l'historien est tout disposé à convenir, tant les textes abondent alors qui en portent la marque. Certains soulignent, à mi-mots, le devoir particulier de contention que la plage fait à l'homme, saisi qu'il est de mille sollicitations émoustillantes ; ils disent l'interdiction des regards trop appuyés ou l'escamotage des érections naissantes. D'autres, en nombre, se proposent d'enseigner aux femmes la nouvelle grammaire balnéaire des rapports à soi ; ils les engagent à se rendre maîtresses des formes d'aisance inédites que réclament le fait de se montrer à demi nue sur la plage, à savoir comment marcher ou se tenir ; ils codifient avec soin les précautions dont doivent s'entourer les rites du déshabillage public, ce moment périlleux où se négocie le passage d'un état (civilisé) en un autre (ensauvagé). "Même si vous avez un maillot de bain dessous, attention à la façon dont vous vous déshabillez. La robe boutonnée devant: oui. La robe qu'on enlève par la tête : non!”

Seulement voilà : ces belles explications n'expliquent pas tout. Oublieuses des luttes et des violences qui tenaillent ce processus, elles passent sous silence le difficile travail de pacification dont la plage est justement l'objet en ces années, et qui mobilise, excusez du peu, l'Église, les maires, les préfets et l'État (Granger, 20।0). Les histoires qu'on se raconte portent ainsi à faire comme si tous ces effets de civilisation, ces déshabillages, ces postures, ces effleurements du regard n'avaient rien à voir avec les lieux particuliers où ils prennent forme et qui, justement, les rendent possibles. Or ce qui est en jeu, dans les batailles qui émaillent le rivage français dans l'entre-deux-guerres, ce n'est pas seulement la lutte pour le contrôle de ce qui se passe sur la plage. Discipliner les usages balnéaires, réglementer la dénudation ou la façon de marcher, c'est, nul besoin d'être grand clerc, se réserver le monopole des usages légitimes du lieu. Non, ce qui est en jeu, c'est ni plus ni moins la lutte pour la définition de ce qu'est la plage. Et de ce qu'elle n'est pas. Une lutte intense et inédite, livrée au nom de la morale, on pouvait s'y attendre, mais aussi du droit dont les démocraties sont faites.

Et c'est bien l'essentiel de ce que raconte cette histoire. Ce qui obsède les croisés de la "plage propre", ce n'est pas tant l'invasion des corps dénudés et lascivement exposés. C'est bien plutôt le mouvement qui, de la plage, fait alors un territoire à part, détaché du reste des lieux et vidé des règles usuelles qui les organisent, sans passé ni avenir, rendu à la seule vérité de l'instant. Et les arrêtés municipaux qui couronnent leur lutte sont, au fond, à lire en ce sens : sur ces espaces qui prétendent si farouchement s'en passer, ils inscrivent une norme qui, par son existence même, rattache ces lieux au reste des territoires régulés de longue date. Bref, ces tumultes de plage sont avant tout lutte contre l'autonomisation du territoire balnéaire telle qu'elle se joue alors. Et l'argumentaire déployé par leurs animateurs en témoigne. Pour commencer, séparant avec soin les "plages de famille" des "plages mondaines", distinguant les "bonnes" des "mauvaises" plages, dont ils n'hésitent pas à dresser la liste, et invoquant sans cesse la force des habitudes qui les rattache à leur plage, ils prennent soin d'aller contre la figure métonymique de la plage, la plage, au singulier, ce territoire objet couvert d'un nom commun 
qui n'avait à peu près aucun sens générique avant-guerre, et qui autorise alors, sur la scène publique, la formulation d'un genre bien particulier de conduites qui, parce qu'elles ne peuvent, à proprement parler, avoir lieu qu'ici, lestent en retour la plage d'une évidence nouvelle, avec ses seuils, ses scènes et ses frontières, et dont la consistance se lit au moins autant dans l'ébattement si particulier des corps que dans l'aménagement de l'estran.

Mais il faut pousser l'analyse plus loin. Dans les luttes de plage obstinées qui émaillent le littoral des années 1920-1930, se tient ce que, faute de mieux, on peut appeler un refus de frontière - si l'on admet, avec Georg Simmel, par exemple, que "la frontière n'est pas un fait spatial avec des conséquences sociologiques, mais un fait sociologique qui prend forme spatiale” (Simmel, 1999). À en juger par le zèle que les défenseurs de la morale balnéaire mettent à s'y opposer, cette affaire de frontière est décisive. La plage, pour eux, n'est pas, ne doit pas et ne peut pas être un ailleurs radical. Qu'elle dépayse gentiment les habitudes : très bien (c'est ce qu'elle fait, au fond, depuis le milieu du XIX siècle). Qu'elle prétende les abolir : voilà l'intolérable. Sur ce territoire de vie, comme sur tous les autres, doit se conserver la formalité sociale des conduites, doivent se prolonger les convenances et les conventions qui règlent ce qui se fait et ce que l'on fait. Les mots qui circulent, textes, tracts, pétitions, empreints souvent de morale chrétienne, n'ont de cesse de l'affirmer : une plage digne de ce nom réclame, de ceux qui la peuplent, un devoir de constance ; elle doit être le lieu, non pas d'un relâchement qui donnerait sens à ce qui s'y joue, mais d'une farouche pérennité des rôles, sociaux, familiaux, sexuels, et à travers eux d'un maintien de l'ordre habituel des manières d'être. Sur la plage, exhorte une série d'affiches placardées à l'été 1934, les hommes doivent demeurer attachés à leur rôle de "chef", et les femmes veiller à préserver "[leur] dignité de mère, [leur] honneur de femme, [leur] réputation de Française, [leur] noblesse de chrétienne”. Rien n'est mieux récusé, alors, que ces époux, ces femmes, ces parents qui, une fois gagnée la plage, oublient ce qu'ils sont, se dénudent, s'ébattent comme des enfants, en un mot se relâchent, et s'exposent ainsi, une fois rendus à leur existence ordinaire, à ne jamais retrouver les rapports que réclame la vie de famille. "Quel respect peuvent désormais avoir l'un pour l'autre ces animaux à face humaine et à langage articulé ?” (Pradel, 1935).

C'est dire que cette bruyante hostilité, oubliée depuis d'avoir échoué à faire valoir ses positions, rend visible le processus qui, en ces années, réinvente la plage, lui donne, à travers la mise en jeu particulière des corps qu'elle autorise, des propriétés bien à elle, et en fait, quelles que soient ses dispositions matérielles, un territoire de l'instant. Un territoire liminal, clos sur lui-même, et où tout ce qui se joue se réduit aux circonstances présentes, où s'abolissent les règles, les appartenances et les hiérarchies ordinaires. Un territoire soigneusement arraché au reste des lieux de vie et qui, autrement dit, comme la fête pour Durkheim ou le "moment critique" pour Bourdieu (1988), agit à la manière d'un diapason : de la multitude infiniment disparate des conduites, des attentes, des groupes sociaux et des situations de vie dont palpite d'ordinaire le monde social, la plage fait un horizon commun, ordonné aux mêmes repères, mesuré aux mêmes pratiques, synchronisé en un même présent, propice au miracle des rencontres et des fraternisations les plus improbables. À l'historien, du coup, il ne suffit pas de décrire la naissance du bronzage ou l'émerveillement neuf des chairs exposées sur le rivage pour espérer comprendre ce qui se joue alors ; il lui faut repérer les formes et les raisons d'être du processus d'autonomisation du territoire balnéaire qui, à ce moment précis, rend possible l'émergence de ces usages d'un nouveau genre et puise en eux l'évidence commune de sa réalisation. Or, et la chose mériterait d'être prolongée dans des termes qui ne se borneraient pas aux hétérotopies de Foucault, ce processus à l'œuvre, rendu lisible par les résistances viscérales qu'il suscite, ne se comprend qu'à condition de voir qu'il est bien de son temps. Que cette découpe de la plage et de ce qui y prend vie s'accorde aux palpitations historiques d'une société littéralement obsédée par le brouillage des repères sociaux, fascinée sans cesse par la perte supposée des repères née de la 
guerre et par la recherche de lieux, de fragments de lieux même, où, dans le repli passager des appartenances convenues, peut se refonder le sens de l'individu.

On ne peut manquer d'être saisi, en effet, par l'émerveillement toujours mêlé de luttes, qu'exercent alors, pour n'en retenir que quelquesuns, des lieux d'indiscipline comme le stade, la salle de cinéma ou le hall d'hôtel (Granger, 2011 ; Kracauer, 2008), qui ont bel et bien en commun de rendre possible, pour un moment, et dans des conditions précises, une existence provisoire et refaite de neuf ${ }^{(6)}$. En un mot : la plage, pièce d'une cartographie des minuscules lieux d'écart, incarne à merveille ces territoires de la modernité dont l'entre-deux-guerres cherche partout la réalisation. Et voilà peut-être bien la leçon de cette petite exploration. Pour peu qu'on daigne ne pas les regarder d'en haut, comme l'arrièregarde grincheuse d'un monde qui refuserait de passer la main, les batailles de plage des années 19201930 se montrent d'une étonnante volubilité. À leur façon, elles mettent sur la piste d'une redéfinition de la plage des logiques sociales dont elle est pleine, et du moment historique particulier où elle s'opère. Mais elles sont aussi porteuses, il nous semble, d'une vertu cognitive. Suivre ces luttes, les rendre à leur vitalité oubliée, c'est faire voler en éclats l'illusion des généalogies tracées de mains sûres. C'est introduire des trous, des saccades, des ruptures, dans le grand panorama historique qui prétend conduire aux plages qui nous sont familières. Bref, c'est prendre acte de la profuse diversité des passés balnéaires et renoncer à croire qu'il est encore possible d'écrire une histoire unifiée et apaisée de la plage.

\section{NoTES}

(I) On ne saurait trop méditer, même à plus de vingt ans de distance, les courtes mais précieuses "Considérations de méthode" qui viennent clore ce livre (Corbin, 1988, p. 321-322) ; voir aussi, plus récemment, la mise au point d'ensemble dans Corbin (200I ; 2010, pp. 630-632). Pour la période suivante, voir l'étude de Rob Shields (1991), l'ouvrage classique de Jean-Didier Urbain (1994), et aussi, plus récemment, quoique selon une perspective très différente, la généalogie anglo-saxonne proposée par John Mack (20 I I).

(2) Sur la reconstitution de cet épisode, voir Granger (2009).

(3) Sur l'inscription de cette lutte en tête des résolutions officielles de la Fédération française contre l'immoralité publique, on consultera en particulier le Bulletin d'informations antipornographiques, $n^{\circ} 94$, avril 1933. Et, plus largement, sur le travail de fabrication de la "lutte contre l'immoralité des plages", comme de toutes les luttes morales de ce genre (Walker, 1991), tout spécialement sur le travail de mobilisation conduit en la matière par Émile Pourésy depuis Bordeaux, voir en particulier Granger (2008).

(4) On peut se faire une idée assez précise de la composition sociale de la Fédération française contre l'immoralité publique, et à travers elle une idée moins nébuleuse des milieux dans lesquels s'enracine cette mobilisation nationale, à la lecture des listes de membres régulièrement publiées dans le Bulletin d'informations antipornographiques, d'une part, et en lisant, d'autre part, les mémoires d'Émile Pourésy, secrétaire de cette fédération et infatigable "balayeur des rues", qui fut l'un des principaux acteurs de cette lutte (Pourésy, 1928).

(5) II n'est sans doute pas mauvais de rappeler ici que les qualifications juridiques (attentat à la pudeur, nudisme, etc.) invoquées dans cette lutte à des fins de mobilisation n'ont, en ces années, aucun fondement judiciaire digne de ce nom ; et que, par ailleurs, s'impose alors, jusque devant les tribunaux, une lecture libérale de la "pudeur normale" et des motifs qui peuvent venir l'outrager (Hugueney, 1938).

(6) Ces lieux d'indiscipline dont il faudrait poursuivre l'analyse croisée, à coup sûr, mais dont on devine assez que, devenus centraux en ces décennies, ils conduisent loin des "lieux de discipline" que constituaient, au siècle précédent, la caserne, l'école ou la prison (Foucault, 1975). 


\section{Références bibliographiques}

Georges ANQUETL, Le Bal sur le volcan. Mœurs de vacances, Anquetil, 1927.

Jean BERNARD, De l'utilisation du domaine public maritime. Établissements de pêche et locations de plage, thèse de droit, université de Paris, 1930.

Patrick BOUCHERON, L'Entretemps. Conversations sur l'histoire, Verdier, 2012.

Pierre Bourdieu, Homo academicus, Minuit, 1988 [1984]

Christophe CHARLE, La Crise des sociétés impériales. Allemagne, France, Grande-Bretagne, 1900-1940. Essai d'histoire sociale comparée, Seuil, 2001.

Alain Corbin, Le Territoire du vide. L'Occident et le désir du rivage, 1750-1840, Aubier, 1988.

Alain CoRBIN, L'Homme dans le paysage, Textuel, 2001. Alain CORBIN, "Plage", dans Christian Delporte, Jean-Yves Mollier et Jean-François Sirinelli (dir.), Dictionnaire d'histoire culturelle de la France contemporaine, Puf, 2010.

Norbert ELIAS, La Civilisation des mœurs, Calmann-Lévy, 1973

(Ire édition 1939)

Michel FouCAULT, Surveiller et punir. Naissance de la prison, Gallimard, 1975.

Christophe Granger, "Batailles de plage. Nudité et pudeur dans l'entre-deux-guerres", Rives méditerranéennes, n 30, juin 2008.

Christophe Granger, Les Corps d'été. Naissance d'une variation saisonnière, Autrement, 2009.

Christophe Granger, "Du relâchement des mœurs en régime tempéré. Corps et civilisation dans l'entre-deux-guerres" (numéro spécial: "Norbert Elias et le 20 siècle. Le processus de civilisation à l'épreuve'), Vingtième siècle. Revue d'histoire, $n^{\circ} 106$ 2010.

Christophe GranGER, "Les lumières du stade. Football et goût du spectaculaire dans l'entre-deux-guerres", Sociétés \&

Représentations, n 31, 2011.

Louis HugueneY, "Crimes et délits contre les particuliers nudité constitutive d'outrage public à la pudeur', Revue de science criminelle et de droit pénal comparé, avril-juin 1938.

Siegfried Kracauer, "Le hall d'hôtel", dans L'Omement de la masse. Essai sur la modemité weimarienne, La Découverte, 2008

(Ire publication 1963)

Jean-Yves LE NAOUR, "Un mouvement antipornographique : la Ligue pour le relèvement de la moralité publique (1883-1946)", Histoire, économie et société, n 3, 2003.

Remi LENOIR, Généalogie de la morale familiale, Seuil, 2003. John MAck, The Sea. A Cutural History, Reaktion Books, 201 I. Philip NORD, "Catholic culture in Interwar France", French Politics, Culture and Society, $n^{\circ}$ 3, 2003

Pascal ORY, L'Invention du bronzage. Essai d'une histoire culturelle, éditions Complexe, 2008

Georges Perec, Espèces d'espaces, Galilée, 2000 (Ire édition 1974).

Émile PouRÉsY, Souvenirs de vingt-cinq années de lutte contre l'immoralité publique, Bordeaux, 1928

Henri Pradel, "La lutte contre l'immoralité des plages", dans Les Devoirs de vacances des parents, Desclée de Brouwer, 1935 Jean-Paul Scot, "La crise sociale des années 1930 en France. Tendances et contre-tendances dans les rapports sociaux", Le Mouvement social, $n^{\circ}$ |42, 1988.

Rob SHIELDS, Places on the Margin. Alternative Geographies of Modernity, Routledge, 1991.

Georg SimmeL, Sociologie. Étude sur les formes de la socialisation, Puf, 1999.

Jean-Didier URBAIN, Sur la plage. Mœurs et coutumes balnéaires, XIXe-XXe siècles, Payot, 1994.

Georges Vigarello, Histoire de la beauté. Le corps et l'art d'embellir de la Renaissance à nos jours, Seuil, 2004

Johan VINCENT, L'Intrusion balnéaire. Les populations littorales bre tonnes et vendéennes face au tourisme, Presses universitaires de Rennes, 2007

Jack L. WALKER, Mobilizing Interest Groups in America. Patrons, Professions, and Social Movements, University of Michigan Press, 1991.

Jessica WARDHAUGH, "Sacred Unions : Religion and Reconstruction in French Society, 1919-1945", French Politics, Culture and Society, $n^{\circ}$ 3, 2009. 